Kurbanov R.D., Mullabaeva G.U.

MODERN OPPORTUNITIES OF PRIMARY PROPHYLAXIS OF SUDDEN CORONARY DEATH FROM A POSITION OF EARLY CORRECTION OF ACTIVITY THE NEUROHORMONAL SYSTEMS AT PATIENTS WITH MYOCARDIAL INFARCTION

The Republican Specialized Center of Cardiology, Tashkent, Uzbekistan

\section{PEЗЮME}

Статья посвящена первичной профилактике внезапной сердечной смерти путем воздействия на ренин-ангиотензин-альдостероновую и симпатоадреналовую системы. Приведены результаты многочисленных трайлов с использованием бета-блокаторов и ингибиторов ангиотензинпревращающего фермента. Обосновано как можно раннее использование этих групп препаратов у пациентов с острым инфарктом миокарда.

Ключевые слова: ишемическая болезнь сердца, внезапная коронарная смерть, симпатоадреналовая и ренин-ангиотензин-альдостероновая система, бета-блокаторы, ингибиторы АПФ.

\section{SUMMARY}

The article is devoted to primary prophylaxis of sudden cardiac death by influence on renninangiotensin-aldosterone and sympathetic systems. Results numerous trials with use beta-blockers, and ACE inhibitors are resulted. It is proved as early use of these groups of preparations at patients with myocardial infarction is possible.

Keywords: ischemic heart disease, sudden coronary death, sympathetic and renin-angiotenzin aldosterone system, beta-blockers, ACE inhibitors

\title{
Контактная информация:
}

Курбанов Равшанбек Давлетович Республиканский Специализированный Центр Кардиологии, г. Ташкент, Узбекистан. Директор. Тел +998712373816

Муллабаева Гузаль Учкуновна Республиканский Специализированный Центр Кардиологии, г. Ташкент, Узбекистан. Лаборатория Аритмий Сердца. Старший научный сотрудник. Контактные тел +998973338683.

Индекс 100052 Узбекистан, г.Ташкент, ул.Осие, 4. Республиканский Специализированный Центр Кардиологии. Факс +998712341667 Guzal-m@inbox.ru УДК 616.127-005.8-036.3/.4-037 
В экономически развитых странах ежедневно внезапно умирает около 2500 человек, причем, только в 2-5\% случаев смерть наступает в медицинских учреждениях (Бойцов С.А. и соавт. 2011 г). В мире ежегодно около 3 млн. человек умирает внезапно при возможности выживания не более $1 \%$. По данным Американской Ассоциации сердца (2004 г), в течение 6 лет после инфаркта миокарда (ИМ), несмотря на оптимальное лечение, $18 \%$ мужчин и 35\% женщин переносят повторный ИМ, $7 \%$ мужчин и 6\% женщин внезапно умирают, $22 \%$ мужчин и 46\% женщин становятся инвалидами из-за развития тяжелой сердечной недостаточности (CH). Вероятность успешной реанимации внезапно умерших вне стационара даже в экономически развитых странах составляет не более $5 \%$ случаев. На сегодняшний день результаты многочисленных исследований позволили выделить три основных момента, определяющих прогноз жизни больных, перенесших ИМ: резидуальная ишемия миокарда, левожелудочковая дисфункция и электрическая нестабильность миокарда. Электрическая стабильность миокарда и его глобальная сократительная способность являются интегральными характеристиками, определяющими функционирование сердца как саморегулирующейся системы. В понятие стабильности вкладывается способность возвращаться к своему уровню функционирования, несмотря на различные внешние воздействия (Бабский Е.Б., 1980). Диагностикой нарушений ритма сердца нельзя ограничиться при оценке степени электрической нестабильности, она - составная её часть, но и только. Единого общепринятого определения понятия электрической нестабильности сердца (ЭНС) не существует до настоящего времени. Более того, различными исследователями (Гавриш А.С., Сергиенко О.В. и соавт., 1997, Hallstrom A.P., Cobb L.A. et al., 1986, Brugada P., Wellens H.J., 1988, Braunwald E., 2001) в понятие ЭНС вкладываются совершенно разные представления. В нашем представлении электрическая нестабильность миокарда это стереотипная реакция сердца при воздействии на него различных патогенных факторов, заключающаяся в существенном изменении электрофизиологических свойств сердечной мышцы, проявляющаяся клинически нарушениями ритма и прогностически характеризующаяся различной степенью риска развития фатальных кардиальных событий. Это определение подтверждается и доказано взаимосвязью между структурной перестройкой миокарда, так называемым постинфарктным ремоделированием (ПИР), и выявляемостью желудочковых нарушений ритма сердца [1]. Согласно результатам многоцентрового исследования MUSTT, фракция выброса и желудочковые тахиаритмии, индуцируемые при электрофизиологическом исследовании, позволяют идентифицировать пациентов с высоким риском смерти. При этом сократимость не коррелирует с каким-либо определенным видом смертности, в то время как нарушения ритма, особенно в сочетании с фракцией выброса $<30 \%$, с высокой долей вероятности позволяют предположить аритмическую ее причину.

Так, по нашим собственным данным, полученным на основании результатов наблюдения 115 больных мужского пола, перенесших ИМ, группа пациентов с желудочковыми аритмиями (ЖА) высоких градаций, выявленных через 2 месяца от начала заболевания, характеризовалась достоверно большим конечно-диастолическим размером (КДО) и низкой фракцией выброса (ФВ). Кроме того, при анализе влияния ЖА на постинфарктный прогноз нами выявлена еще одна особенность, на которую следует обратить внимание. Так оказалось, что наличие частой и сложной ЖА имело достоверную связь не только с летальностью, но и с высокой вероятностью развития или прогрессирования в последующем $\mathrm{CH}(8,0$ против $1,9 \%$ у больных без ЖА, Х2 - 8,66, $\mathrm{p}<0,05)$. Подтверждением прямой зависимости желудочковой эктопической активности от размеров поражения миокарда являются результаты изучения частоты и характера ЖА при различной кратности локализации и размеров ИМ. Так, ЖА, в т.ч. ее высокие градации при повторном, передне-нижнем и Q-ИМ выявлялись достоверно чаще, чем соответственно при первичном, переднем, нижнем и без Q-ИМ. Таким образом, полученные результаты свидетельствуют о тесной взаимозависимости ЖА и нарушения сократительной способности миокарда, что совпадает с литературными данными. Из этого следует, что, во-первых, появление и сохранение частой ЖА через 2 месяца после ИМ является косвенным признаком ПИР миокарда и связанного с ним нарушения сократительной способности; во-вторых, наличие желудочковой экстрасистолии (ЖЭ) высоких градаций (политопной, парной и/или групповой) может быть использовано в качестве маркера высокой вероятности смертельного исхода и, в третьих, маловероятно, что прогноз жизни данного контингента может быть улучшено только за счет антиаритмической терапии (AАT).

Так, исследованиями CAST и ESVEM, в которых использовались антиаритмические препараты (ААП) I класса было отвергнуто предположение, что устранение аритмии способствует снижению риска внезапной коронарной смерти (ВКС). Более 
того, анализ исследований позволил выделить группу препаратов, повышающих частоту аритмических событий.

Несмотря на успехи применения имплантируемых кардиовертеров-дефибрилляторов (ИКВДФ) в крупных исследованиях (MADIT, AVID, GABGPatch, MUSTT, CASH) у больных, переживших остановку сердца, их эффективность в качестве средств первичной профилактики недостаточно доказана. К тому же в нашей стране они пока малодоступны в силу высокой стоимости. Кроме того, после имплантации КВДФ в 70\% случаев приходится назначать ААП [2]. У 50\% больных это делается для снижения частоты рецидивирования ЖТ, что снижает частоту включения ИКДФ и продлевает его «жизнь» [3].

Препарат III группы амиодарон доказал свою ААЭ в отношении ЖА. Ряд исследований, проведенных у больных, перенесших ИМ (BASIS, Polish Amiodarone Trial, Spanish Study of Sudden Death, EMIAT, CAMIAT), доказали его способность снижать риск ВКС и общую летальность у больных, перенесших ИМ [4]. Отсутствие эффекта одних ААП и наличие его у амиодарона возможно обусловлено его влиянием на такие патогенетические звенья ВКС, как резидуальная ишемия, ЭНС, утрата «парасимпатической защиты».

Бета-адреноблокаторы значительно уменьшают частоту общей и BKC $[5,6]$. Их положительное влияние объясняется не только антиаритмическим эффектом, как показало исследование MUSTT [7], но и уменьшением зоны ишемии, снижением ЧСС, поддержанием должного уровня автономного баланса. Предполагается, что бетаблокаторы за счет уменьшения симпатических влияний значимо и достоверно снижают альтернацию волны Т у пациентов с ИБС и левожелудочковой дисфункцией [8]. Исследование AVID продемонстрировало, что у пациентов с жизнеугрожающими ЖА при невозможности имплантации ИКВДФ и терапии амиодароном, бета-блокаторы достоверно улучшают выживаемость [9].

Положительное влияние иАПФ на выживаемость больных после перенесенного ИМ и отсутствие у них при этом прямого ААЭ, способствовали совершенствованию патогенетической терапии ИМ с упрочением значения ПИР.

Причинно-следственные связи, лежащие в основе процесса ПИР, активно изучаются. В качестве основных детерминант, определяющих характер ПИР рассматриваются размер, глубина и локализация некроза, объем нефункционирующего жизнеспособного миокарда, проходимость инфаркт-связанной артерии, общая выражен- ность атеросклеротического поражения коронарных сосудов. Влияние этих факторов во многом опосредуется развивающимся дисбалансом различных нейрогуморальных систем, в первую очередь, симпато-адреналовой (САC) и ренинангиотензин-альдостероновой (РААС). Выдвинута гипотеза, что возникающая нейрогуморальная активация не только является следствием нарушения насосной функции сердца и зависит от его тяжести, но и сама способствует прогрессирующему ремоделированию миокарда и сосудов, во многом формируя морфологический субстрат синдрома $\mathrm{CH}$, и оказывает непосредственное влияние на течение заболевания [10]. Так, гиперактивность САС и РААС в сочетании с депрессией барорефлекторной чувствительности является независимым предиктором плохого прогноза у больных СН [11]. Кроме того, гиперсимпатикотония может явиться причиной локальной аккумуляции дериватов норадреналина, обладающих прооксидантными свойствами, и, таким образом, модулировать оксидантный стресс с высвобождением ряда нейротрансмиттеров, обладающих цитотоксическим действием [12].

Ранняя активация РАС после ИМ и ее вовлечение в патогенез СН свидетельствуют в пользу введения иАПФ в схему лечения больных ИМ. Роль иАПФ, примененных в ранние сроки заболевания, изучалась в нескольких крупномасштабных клинических исследованиях. В исследованиях с назначением иАПФ перорально установлено снижение смертности и частоты развития осложнений у всех больных с ИМ, включая обследованных с подозрением на него. Указанные исследования можно разделить на 2 группы: с ранним (CONSENSUS-2, CCS-1, GISSI-3, ISIS-4, SMILE) [13, 14, $15,16]$ и относительно поздним (SAVE, AIRE, TRACE) назначением препарата $[16,17,18]$. Достоверное положительное влияние на смертность в ближайший месяц было отмечено только в наиболее крупных исследованиях: GISSI-3, ISIS-4. В исследованиях CCS-1, SMILE установлена аналогичная тенденция, но различия между группами были статистически недостоверными, а в отношении смертности стали достоверными спустя 1 год. В исследовании CONSENSUS-2 отмечена тенденция к увеличению смертности в группе больных, получавших иАПФ, что стало причиной преждевременного прекращения испытания [13]. Большинство экспертов утверждают, что определенную роль сыграла особенность использования иАПФ (первоначальное внутривенное введение фиксированной дозы длительно действующего препарата), способствовавшая частому возникновению 
артериальной гипотензии, особенно у пожилых. Вследствие этого внутривенное введение иАПФ в первые 24 ч у больных ИМ с подъемом сегмента ST на ЭКГ в настоящее время считается противопоказанным из-за угрозы артериальной гипотензии [20]. Крупное рандомизированное плацебоконтролируемое исследование SMILE-I (Survival of Myocardial Infarction Long-Term Evaluation) одно из немногих, в котором убедительно продемонстрирована польза раннего начала терапии иАПФ (зофеноприл) при остром ИМ без клинических проявлений СН [21]. Успех исследования в значительной мере определяется выбором такой категории больных острым ИМ, у которых эта терапия особенно оправдана, а именно, у больных с ИМ передней стенки ЛЖ, которые не получали тромболитическую терапию (наличие противопоказаний, позднее поступление в палату интенсивной терапии или ИМ без стойких подъемов сегмента ST).

Однако, с другой стороны, задержка начала приема иАПФ (в пределах 24-36 после начала ИМ) заметно не сказывалась на их влиянии на смертность в ближайшие 30 сут, что указывает на возможность отсроченного назначения препаратов этой группы в случаях, когда в ранние сроки заболевания существуют относительные противопоказания для начала титрования дозы. ИАПФ следует назначать всем больным ИМ независимо от возраста, сроков заболевания, проводимого лечения и степени риска развития осложнений, однако выгода от клинического их применения выше у больных с передней локализацией ИМ, признаками сердечной недостаточности и пониженной функцией ЛЖ.

Если говорить об осложнениях ИМ, то хотелось бы вернуться к вышеописанным ЖА, которые при сочетании (а чаще всего это сочетание закономерно) с передней локализацией ИМ, сниженной функцией ЛЖ без сомнения формируют высокий риск развития ВКС. Конечно, естественным и логичным было бы предположить, что своевременное и адекватное назначение антиаритмических препаратов, имеющихся сегодня в нашем арсенале будет способствовать снижению этого риска. Так, в книге H.E. Kulbertus и H.J.J.Wellens «Первый год после инфаркта миокарда» указывается, что возникновение первого пароксизма желудочковой тахикардии (ЖТ) вне острой фазы заболевания, но в пределах первых 8 недель, при отсутствии антиаритмической терапии или ее случайном назначении сопряжено с $83 \%$ летальностью в течение 1 года. Половина переживших этот срок больных умирают в течение следующих 3 лет. Но необходимо напомнить, что генез арит- мии у больных ИМ в первую очередь определяется снижением насосной функции сердца. Развивающаяся в ответ гиперсимпатикотония является непосредственной причиной возникновения фатальных ЖА в условиях ЭНС [22]. Необходимо сказать и о выраженном проаритмическом эффекте ангиотензина II. Все эти факторы и объясняют положительный эффект иАПФ по снижению смертности у больных перенесенным ИМ [23].

Исследование CARMEN (Carvedilol ACE Inhibitor Remodelling Mild HF Evaluation, или «Исследование влияния карведилола и ингибитора АПФ на ремоделирование при легкой форме сердечной недостаточности») - первое крупномасштабное исследование, в котором непосредственно сравнивали влияние бета-блокады и ингибирования АПФ на функцию ЛЖ при ХСН. Контролируемое, проспективное, двойное слепое, рандомизированное исследование было запланировано для изучения влияния комбинации ингибитора АПФ (эналаприла) с дилатрендом, в сравнении с монотерапией эналаприлом или дилатрендом, на ремоделирование у больных с легкой формой ХCH. Главной конечной точкой исследования было среднее изменение индекса конечно-систолического объема ЛЖ (иКСО ЛЖ). Его определяли как адекватный показатель ремоделирования исходно и в динамике, к 18-му месяцу (окончание поддерживающей фазы лечения), сравнивая группы комбинированной терапии дилатрендом и эналаприлом с группами монотерапии дилатрендом либо эналаприлом. В исследование CARMEN вошли 572 пациента с легкой ХСН из 65 центров 13 европейских стран. В анамнезе у больных отмечалось наличие легких симптомов XСН в течение не менее 2 месяцев на фоне терапии, не менявшейся в течение 2 недель до исходного визита. Другими критериями, необходимыми для включения в исследование, были исходная фракция выброса левого желудочка < $39 \%$ (оцененная с помощью эхокардиографии) и двухнедельный период, считая от исходного визита, без неотложных госпитализаций по сердечно-сосудистым причинам. Больные с симптомами в покое, с нестабильной XCH или требующие реваскуляризации сердца, исключались из исследования. Комбинированная терапия дилатрендом и эналаприлом привела к высоко достоверному уменьшению иКСО ЛЖ, по сравнению с монотерапией эналаприлом $(p<0,002)$, с обратным развитием ремоделирования ЛЖ уже через 6 месяцев, сохранявшимся в течение всего периода лечения. Монотерапия дилатрендом приводила к достоверному обратному развитию ремоделирования, по сравнению с исходным $(p<0,05)$. На монотерапии эналаприлом 
подобного результата получено не было. Все три терапевтические группы продемонстрировали очень сходный хороший профиль переносимости и безопасности, и, вопреки распространенному мнению о худшей переносимости бетаблокаторов, дилатренд нисколько не уступал по переносимости ингибитору АПФ. Действительно, дилатренд был без проблем назначен до начала приема ингибитора АПФ. Частота выбывания пациентов из трех терапевтических групп была сходной. Примечательно, что данные по смертности и госпитализациям в трех терапевтических группах также были одинаковыми. Исследование CARMEN подтверждает правильность современных рекомендаций по лечению ХСН и обосновывает раннее назначение комбинированной терапии дилатрендом и ингибитором АПФ больным с легкой степенью ХCH.

Опираясь на данные собственного наблюдения, мы можем подтвердить, что раннее назначение этих основных двух групп препаратов у больных, перенесших Q-волновой ИМ, способствовало замедлению патологического ПИР и выявляемости ЖА высоких градаций. Так, нами были ретроспективно изучены данные по 123 пациентам, которые находились на стационарном лечении в Республиканском Центре Кардиологии республики Узбекистан в 1991-1995 (57 больных) и 2003-2005 (66 больных) гг. Пациенты обеих групп были сопоставимы по демографическим и анамнестическим данным, а также по характеру течения ИМ (табл.1).
При ХМ ЭКГ, проведенном на 10-14 сутки заболевания, частая ЖЭ и ЖЭ высоких градаций достоверно чаще регистрировалась в группе больных, обследованных в 1991-1995 гг (57\% против $33 \%, X 2=4,62 ; p=0,03)$. При структурном анализе ЖЭ нами выявлено, что прогностически неблагоприятные ЖЭ (IV A класc по Lown) в 2 раза чаще встречалась в группе пациентов, находившихся на стационарном лечении в 1991-1995 гг. (21\% против $10 \%, X 2=7,68 ; p=0,005)$. Необходимо отметить, что ЖЭ IV6 и V классов на предвыписном этапе за 2002-2005 гг.не выявлялись.

Через 6 месяцев от начала ИМ при проведении ХМ ЭКГ выявлены достоверные различия между группами по числу больных, имеющих неблагоприятные виды ЖЭ: в 1991-1995 гг. их было больше, чем в группе сравнения $(P=0,03)$. Индивидуальный анализ динамики ЖЭ показал, что в группе больных, обследованных в 1991-1995 гг. при повторном ХМ ЭКГ (через 6 мес. от начала заболевания) неблагоприятные виды ЖЭ перестали регистрироваться только в 2,4\% случаях, тогда как в группе сравнения количество пациентов с ЖЭ уменьшилось вдвое $(X 2=12,7 ; \mathrm{p}=0,001)$; уменьшение максимальной градации как минимум на 1 пункт отмечено у 4,8 и 20,5\% (2 и 8) больных $(\mathrm{P}=0,04)$, а такое же увеличение у 16,7 и $5,1 \%$ (7 и 2) больных соответственно $(\mathrm{P}=0,10)$. В то же время в первой группе появились новые случаи ЖЭ. Так, в 1991-1995 гг. они составили 9,5\%. Процесс ПИР ЛЖ оценивался по его конечному диастолическому размеру (табл.2).

Табл. 1. Структура ЖА по данным ХМЭКГ проведенного в различные сроки ОИМ (оценка по максимальной градации ЖЭ)

\begin{tabular}{|c|c|c|c|c|c|c|}
\hline \multirow[t]{2}{*}{ Градация } & \multicolumn{3}{|c|}{ Перед выпиской из стационара } & \multicolumn{3}{|c|}{ Через 6 мес от начала ОИМ } \\
\hline & $\begin{array}{l}\text { 1991-1995гr } \\
\text { (18-21день) }\end{array}$ & $\begin{array}{l}\text { 2002-2005rг } \\
\text { (10-14 день) }\end{array}$ & $\mathbf{p}$ & $\begin{array}{l}\text { 1991-1995rr } \\
(6 \mathrm{mec})\end{array}$ & $\begin{array}{l}2002-2005 \mathrm{rr} \\
(6 \mathrm{mec})\end{array}$ & $\mathbf{p}$ \\
\hline >10 ЖЭ & $4(9,5 \%)$ & $5(12,8 \%)$ & $\begin{array}{c}X^{2}=2,18 \\
p=0,14\end{array}$ & $4(9,5 \%)$ & $3(7,7 \%)$ & $\begin{aligned} X^{2} & =3,64 ; \\
p & =0,05\end{aligned}$ \\
\hline 3 & $4(9,5 \%)$ & $4(10 \%)$ & $\begin{aligned} X 2 & =0,99 ; \\
p & =0,32\end{aligned}$ & $4(9,5 \%)$ & $2(5,1 \%)$ & $\begin{aligned} X^{2} & =1,13 ; \\
p & =0,26\end{aligned}$ \\
\hline $4 a$ & $9(21 \%)$ & $4(10 \%)$ & $\begin{array}{l}X^{2}=7,68 \\
p=0,005\end{array}$ & $10(23,8 \%)$ & $1(2,5 \%)$ & $\begin{array}{l}X^{2}=12,5 ; \\
p=0,004\end{array}$ \\
\hline 46 & $6(14,3 \%)$ & - & & $8(19,0 \%)$ & 0 & \\
\hline 5 & $1(2,4 \%)$ & - & & $1(2,4 \%)$ & 0 & \\
\hline Bcero & $24(57 \%)$ & $13(33 \%)$ & $\begin{array}{c}X^{2}=4,62 ; \\
p=0,03\end{array}$ & $27(64,3 \%)$ & $6(15 \%)$ & $\begin{array}{l}X^{2}=12,7 ; \\
p=0,001\end{array}$ \\
\hline
\end{tabular}

ЖА - желудочковая аритмия

ЖЭ-желудочковая экстрасистолия

Использованы непараметрические методы анализа с использованием критерия Х2. Различия или изменения считали достоверными при уровне значимости $p<0,05$ 
Таблица 2 Величина КДР в различные сроки ИМ

\begin{tabular}{|c|c|c|c|c|c|c|c|c|}
\hline \multirow{2}{*}{$\begin{array}{c}\text { Группа } \\
\text { больных }\end{array}$} & \multicolumn{4}{|c|}{ Перед выпиской из стационара } & \multicolumn{4}{|c|}{ Через 6 мес от начала ОИМ } \\
\hline & $\begin{array}{c}\text { n } 1991- \\
1995\end{array}$ & $\begin{array}{c}\text { КДР, мм } \\
(\mathrm{M} \pm \mathrm{SD}) \\
1991- \\
1995 \text { гг }\end{array}$ & $\begin{array}{c}\text { КДР, мм } \\
(\mathrm{M} \pm \mathrm{SD}) \\
2002-2005 \\
\text { гг }\end{array}$ & $\begin{array}{l}\text { n 2002- } \\
2005\end{array}$ & $\begin{array}{c}\text { n 1991- } \\
1995\end{array}$ & $\begin{array}{c}\text { КДР, мм } \\
(\mathrm{M} \pm \mathrm{SD}) \\
1991- \\
1995\end{array}$ & $\begin{array}{c}\text { КДР, мм } \\
(\mathrm{M} \pm \mathrm{SD}) \\
2002- \\
2005 г г\end{array}$ & $\begin{array}{c}\text { n 2002- } \\
2005\end{array}$ \\
\hline Всего & 42 & $5,79+0,81$ & $5,76+0,62$ & 39 & 42 & $5,89+0,74$ & $5,69+0,67$ & 39 \\
\hline $\begin{array}{l}\text { Без } \\
\text { ПОЖА }\end{array}$ & $\begin{array}{c}18 \\
(43 \%)\end{array}$ & $5,59+0,75$ & $5,45+0,52$ & $23(59 \%)$ & $15(36 \%)$ & $5,68+0,63$ & $5,52+0,60$ & $33(85 \%)$ \\
\hline С ПОЖА & $\begin{array}{c}24 \\
(57 \%)\end{array}$ & $5,97+0,71$ & $6,05+0,59$ & $16(41 \%)$ & $27(64 \%)$ & $6,03+0,84$ & $5,86+0,71$ & $6(15 \%)$ \\
\hline $\mathrm{p}-$ & & 0,10 & 0,002 & 0,42 & & 0,126 & 0,156 & \\
\hline
\end{tabular}

КДР-конечно-диастолический размер

Средние значения КДР на предвыписном этапе между двумя группами не различались. Однако имелась отчетливая тенденция к увеличению КДР ЛЖ при наличии частой ЖЭ и ЖЭ высоких градаций внутри групп. В изучаемых группах больных отмечались противоположные тенденции в динамике КДР за 6 мес. наблюдалось некоторое увеличение средне-группового его значения (на 2,7\%), то в 2003-2005гг., напротив, его уменьшение на (на 1,9\%).

Также был проведен сравнительный анализ базисной терапии, полученной в стационаре и на амбулаторном этапе наблюдения вплоть до 6 месяцев от начала ИМ (табл.3).

Полученные данные свидетельствуют о высо- козначимых статистических различиях в частоте назначения больным ИМ иАПФ и статинов как в госпитальной, так и в постгоспитальной стадии заболевания. Число больных, леченых иАПФ, увеличилось в 7 раз, а доля больных, постоянно принимающих статины в 9 раз $(\mathrm{P} \leq 0,0001)$. 3а период 2002-2005 гг. 20,5\% пациентам проведена тромболитическая терапия, тогда как в группе сравнения этот вид вмешательства вообще не использовался. Достоверно увеличилось число пациентов, длительно леченных аспирином $(P=0,023)$. Длительное лечение статинами и аспирином также оказывает благоприятное влияние на процессы ремоделирования и аритмогенеза после ИМ. Их непрямое кардиопротективное

Таблица 3 Характер проводимой терапии в различные сроки ИМ

\begin{tabular}{|l|c|c|c|c|}
\hline Tерапия & $\mathbf{1 9 9 1 - 1 9 9 5 r r ~}$ & $\mathbf{2 0 0 2 - 2 0 0 5 r r ~}$ & Критерий х2 & P-значение \\
\hline Госпитальная стадия ОИМ & \multicolumn{4}{|c|}{} \\
\hline Тромболитическая терапия & 0 & $8(20,5 \%)$ & - & 0,002 \\
\hline Аспирин & $32(76,2 \%)$ & $39(100 \%)$ & - & 0,0008 \\
\hline Бета-блокаторы & $8(42,9 \%)$ & $30(76,9 \%)$ & 8,36 & 0,004 \\
\hline Ингибиторы АПФ & $3(7,1 \%)$ & $19(48,7 \%)$ & 15,63 & 0,0001 \\
\hline Статины & 0 & $16(41 \%)$ & 18,96 & 0,00001 \\
\hline Нитраты & $40(95,2 \%)$ & $38(97 \%)$ & - & 0,53 \\
\hline После выписки из стационара & & & & \\
\hline Аспирин & $31(73,8 \%)$ & $37(94,9 \%)$ & 5,19 & 0,023 \\
\hline Бета-блокаторы, В т.ч. карве- & $21(50 \%) 0$ & $35(89,6 \%)$ & $13,615,8$ & 0,0003 \\
\hline дилол & & $14(35,9 \%)$ & & 0,0001 \\
\hline Ингибиторы АПФ & $4(9,5 \%)$ & $27(69,2 \%)$ & 28,04 & 0,00001 \\
\hline Статины & $3(7,1 \%)$ & $26(66,7 \%)$ & 28,64 & 0,00001 \\
\hline
\end{tabular}

Использованы непараметрические методы анализа с использованием критерия X2. Различия или изменения считали достоверными при уровне значимости $p<0,05$ 
действие опосредуется улучшением реологических свойств крови и эндотелиальной функции коронарных сосудов, оптимизацией перфузии миокарда, разрешением его оглушенности и гибернации, что в конечном итоге приводит к улучшению локальной и глобальной сократимости ЛЖ. В 1,7 раз увеличилось число больных, принимавших бета-адреноблокаторы как в стационаре, так и после выписки $(p<0,005)$, при этом в 2003-2005 гг. более чем в 1/3 случаев назначался карведилол.

Полученные нами результаты свидетельствуют о значительных изменениях в лечении постинфарктных больных, произошедших за последние 10-12 лет. Современная базисная терапия ИМ, несомненно, явилась благоприятным фоном для снижения распространенности ЖЭ. Нами не было получено достоверных различий в динамике КДР в изучаемых группах больных. Вероятно, при выбранной длительности наблюдения, кардиопротективное действие препаратов реализуется в основном на клеточном и субклеточном уровнях. Для получения более весомых результатов для макроскопических структурных параметров ЛЖ требуется увеличение его сроков.

Таким образом, на сегодняшний день терапия с как можно ранним включением групп препаратов (бета-блокаторы, иАПФ), обладающих модулирующим эффектом на нейрогуморальную активность, доказала свою безопасность и эффективность в первичной профилактике ВКС у больных, перенесших ИМ.

Числовые данные представлены в виде $\mathrm{M} \pm \mathrm{SD}$, где M - среднее, SD - стандартное отклонение. Межгрупповые сравнения количественных переменных в 2-х группах проводили с использованием критерия $\mathrm{t}$ Стьюдента. Различия или изменения считали достоверными при уровне значимости $\mathrm{p}<0,05$

\section{СПИСОК ЛИТЕРАТУРЫ}

1. Голицын С.П. Лечение желудочковых аритмий с позичии первичной и вторичной профилактики внезапной смерти. // Сердечная недоcmaточность. - 2001. - T. 2. - № 5. - C. 2 - 10.

2. ACC/AHA Guidelines for the Management of Patients with ST-elevation Myocardial Infarction. A Report of the American College of Cardiology/ American Heart Association Task Force on Practice Guidelines (Committee to Revise the 1999 Guidelines for the Management of Patients With Acute Myocardial Infarction).

3. Borghi C., Bacchelli S., Esposti D.D. et al. SMILE Study. Effects of the early ACE inhibition in diabetic nontrombolyzed patients with anterior acute myocardial infarction // Diabetes Care. - 2003; 26 (6): 1862 - 1868.

4. Brodsky M.A., Mitchell L.B., Halperin B.D.et al. Prognostic value of baseline electrophysiology studies in patients with sustained ventricular tachyarrhythmia: the Antiarrhythmics Versus Implantable Defibrillators (AVID) trial. // Am Heart J, 2002 Sep; 144(3):478-84.

5. Chinese Cardiac Study Collaborative group. Oral Captopril versus placebo among 13634 patients with suspected acute myocardial infarction: interim report from the Chinese Cardiac Study (CCS-1). Lancet 1995; 345:686-687

6. Cohn J., Levine T.B., Olivari H.T. et al. Ejection fraction, exercise consumption, cardiothoracic ration, ventricular arrhythmias, and plasma norepinephrine as determinants of prognosis in heart failure. Circulation 1993; 87: V115 - V116.

7. Effect of ramipril on mortality and morbidity of survivors of acute myocardial infarction with clinical evidence of heart failure. The Acute Infarction Ramipril Efficacy (AIRE) Study Investigators //Lancet. 1993 Oct 2; 342 (8875): 821 $-828$

8. Ellison K.E., Hafley G.E., Hickey K.et al. Effect of betablocking therapy on outcome in the Multicenter UnSustained Tachycardia Trial (MUSTT). // Circulation, 2002 Nov 19; 106(21):2694-9

9. Fletcher R.D., Cintron G.B., Johnson G. et.al. Enalapril decreases prevalence of ventricular tachycardia in patients with chronic congestive failure. Circulation 1993; 87:6:V149 - V155

10. Francis G.S., Cohn J.N., Johnson G. et al. Plasma norepinephrine, rennin activity and congestive heart failure: relations to survival and the effects of therapy in V-HeFT-II. Icid. 1993; 87 (suppl. VI): V140 - V148.

11. Greene L.N. Interactions between pharmacologic and nonpharmacologic antiarrhythmic therapy. // Am. J. Cardiol. - 1996. - Vol. 78 (supp/ 4 A). - P. 61 66.

12. Gruppo Italiano per lo Studio della Sopravvivenza nel' Infarto miocardico-3. GISSI - 3: Effects of lisinopril and transdermal glyceryl trinitrate singly and together on 6-week mortality and ventricular function after myocardial infarction. Lancet 1994:343:1115 - 1122

13. Halawa B. Antiarrhythmic agents in the prevention of sudden cardiac death. // Pol Merkuriusz Lek, 1999 Mar; 6: 117-20.

14. Hilleman D.E., Bauman A.L. Role of antiarrhythmic therapy in patients at risk for sudden cardiac death: an evidence-based review. // Pharmacotherapy, 2001 May; 21 (5): 556-75.

15. ISIS-4 Collaborative Group. ISIS-4: a randomized 
factorial trial assessing early oral captopril, oral mononitrate, and intravenous magnesium sulphate in 58050 patients with suspected myocardial infarction. Lancet 1995; 345:669-685

16. $K$ ber L, Torp-Pedersen C, Carlsen J.E., Bagger H., Eliasen P., Lyngborg K., VidebaekJ., ColeD.S., Auclert L., Pauly N.C. A clinical trial of the angiotenzinconverting-enzyme inhibitor trandolapril in patients with left ventricular dysfunction after myocardial infarction. Trandolapril Cardiac Evaluation (TRACE) Study Group // N Engl J Med. 1995; 333: 1670-1676

17. Masson S., Sesse F., Latini R. et al. Neuroendocrine variability in patients with heart failure. In: 13th World Congress of Cardiology. Rio de Janeiro; 1998. Abstr. 1148.

18. Naccarelli G.V., Wolbrette D.L., Patel H.M., Luck J.C. Amiodarone: clinical trials. /Curr. Opin. Cardiol. 2000. - 16:64 - 72.

19. Podrid P.J., Fuchs T., Candinas R. Role of the sympathetic nervous system in the genesis of ventricular arrhythmia. Circulation 1990; 82:8:1103-1113.

20. Rashba E.J., Cooklin M., MacMurdy K.et al. Effects of selective autonomic blockade on T-wave alternans in humans. // Circulation, 2002 Feb 19; 105 (7): 837-42.

21. Santini M., Cacciatore G., Ansalone G. Heart failure and arrhythmias: the role of ACE inhibitors. Ann Ital Med Int 1993; 8:10S - 13S.

22. Swedberg K., Held P., Kjekhus J. et al for the CONSENSUS II study group. Effects of an early administration of enalapril on mortality in patients with myocardial infarction. Results of the Cooperative New Scandinavian Enalapril Survival Study II. New Engl J Med 1992; 327:678-684

23. Vantrimpont P., Rouleau, Chuan-Chuan W, et al, for the SAVE investigators. Additive beneficial effects of beta-blockers to angiotensin-converting enzyme inhibitors in the Survival and Ventricular Enlargement (SAVE) Study. J Am Coll Cardiol. 1997; 29:229-326 